\title{
Stabilization of solitons in PT models with supersymmetry by periodic management
}

\author{
R. Driben ${ }^{1,2}$ and B. A. MALOMED ${ }^{2}$ \\ 1 Jerusalem College of Engineering, Ramat Beit HaKerem, \\ POB 3566, Jerusalem, 91035, Israel \\ 2 Department of Physical Electronics, School of Electrical Engineering, Faculty of Engineering, Tel Aviv University, \\ Tel Aviv 69978, Israel
}

PACS 11.30.Er - Charge conjugation, parity, time reversal, and other discrete symmetries PACS 42.65.Tg - Optical solitons; nonlinear guided waves

\begin{abstract}
We introduce a system based on dual-core nonlinear waveguides with the balanced gain and loss acting separately in the cores. The system features a "supersymmetry" when the gain and loss are equal to the inter-core coupling. This system admits a variety of exact solutions (we focus on solitons), which are subject to a specific subexponential instability. We demonstrate that the application of a "management", in the form of periodic simultaneous switch of the sign of the gain, loss, and inter-coupling, effectively stabilizes solitons, without destroying the supersymmetry. The management turns the solitons into attractors, for which an attraction basin is identified. The initial amplitude asymmetry and phase mismatch between the components transforms the solitons into quasi-stable breathers.
\end{abstract}

Introduction. Nonlinear dissipative systems subject to the parity-time $(\mathcal{P} \mathcal{T})$ symmetry feature a unique combination of conservative and dissipative properties, the most fundamental one being the real spectrum of eigenvalues generated by non-Hermitian Hamiltonians [1. In the simplest case, such a Hamiltonian contains complex potentials $V(x)$ which obeys the conjugation constraint, $V(x)=V^{*}(-x)$. Recently, it was predicted 2,9 and demonstrated in experiments 10,11 that $\mathcal{P} \mathcal{T}$-symmetric systems can be implemented in optics, making use of symmetric landscapes of the refractive index, combined with antisymmetrically placed linear amplifiers and absorbers, see also recent review 12. The theoretical analysis of $\mathcal{P} \mathcal{T}$ settings that may be implemented in optics was extended by the incorporation of nonlinearities 13 16 (in particular, of cubic gain and loss that are subject to the $\mathcal{P} \mathcal{T}$ invariance condition in its nonlinear form [17,18]). Solitons were predicted in some of these nonlinear systems [19.

A setting which may give rise to a $\mathcal{P} \mathcal{T}$-symmetric system is a Kerr-nonlinear dual-core waveguide, with the gain and loss separately applied to the two cores, which are linearly coupled by the tunneling of electromagnetic waves across the separating barrier. This setting was proposed in Ref. [20] as a medium capable to support stable dissipative solitons. Further analysis has corroborated the existence of one-dimensional solitons in optical $[21,23,25,26]$ and plasmonic 25 versions of the system. Moreover, stable fundamental solitons [27 and vortices 28,29] were predicted in similarly designed dual laser cavities with saturable nonlinearity. The advantage of this scheme in comparison with the ordinary (passive) dual-core systems is that it may be used as the basic element of various lasing setups 23-29. In particular, the PT symmetry can be realized in this dual-core system by balancing the gain and loss in the active and passive linearly-coupled cores. This implies that both cores can be made of a doped lossy material, while the dopant atoms, if pumped externally, may add a linear gain to the system [24]. Then, if the external pump is applied to a single core, it may supply the gain overcompensating the intrinsic loss of this core, so as the net gain provides the $P T$ balance with the other core, that remains lossy. The $P T$-invariant variant of the active system is especially interesting, as it corresponds to the lowest gain level which is capable to stabilize the system.

The dual-core system makes it also possible to define the regime of supersymmetry, with the strength of the linear coupling between the cores equal to the common values of the gain and loss coefficients. It is easy to find 
a broad class of exact solutions for the dual-core system subject to the supersymmetry constraint, including solitons. A challenging issue is the stability of the solitons, as the supersymmetric system is not integrable. In this work, we find a specific subexponential (resonant) instability to which the supersymmetric solitons are subject. Then, we demonstrate that the solitons may be stabilized in the supersymmetric system by means of the management technique 3032 , which periodically switches the gain and loss between the cores, simultaneously reversing the sign of the linear coupling. The former element of the management scheme may be provided by the periodic switch of the core to which the external pump is applied, while the latter one may actually be implemented by periodically shifting the phase of one of the field components by $\pi$, i.e., periodically passing the wave propagating in one core through $\pi$-shifting slabs. The management scheme does not break the supersymmetry, making the solitons stable in a broad parameter region, which is identified by means of systematic simulations. The stabilization provided by this technique complies with the general fact that the management helps stabilize solitons in a variety of waveguiding systems 31, 32 .

The model. The propagation of optical or plasmon waves in the dual-core system obeys the linearly coupled equations for the slow evolution of wave amplitudes $u(Z, T)$ and $v(Z, T)$ in the amplified and damped cores [20- 25]:

$$
\begin{aligned}
i u_{Z}+(1 / 2) u_{T T}+|u|^{2} u-i \gamma u+\kappa v & =0, \\
i v_{Z}+(1 / 2) v_{T T}+|v|^{2} v+i \Gamma v+\kappa u & =0 .
\end{aligned}
$$

Here $Z$ is the propagation distance, $T$ is the reduced time or transverse coordinate in the temporal or spatial system, respectively, $\gamma$ and $\Gamma$ are coefficients of the linear gain and loss acting in the two cores, and $\kappa$ is the strength of the linear coupling between them. The coefficients in front of the group-velocity dispersion (or diffraction) and Kerr terms in Eqs. (1) are scaled to be 1. The $\mathcal{P} \mathcal{T}$-symmetry takes place for $\Gamma=\gamma$, while the supersymmetry condition is $\Gamma=\gamma=\kappa$, which casts Eqs. (1) into the following form:

$$
\begin{aligned}
i u_{Z}+(1 / 2) u_{T T}+|u|^{2} u-i \kappa u+\kappa v & =0, \\
i v_{Z}+(1 / 2) v_{T T}+|v|^{2} v+i \kappa v+\kappa u & =0 .
\end{aligned}
$$

Obviously, substitution $v(Z, T)=i u(Z, T)$ transforms both equation (2) into the standard NLS (nonlinear Schrödinger) equation, $i u_{Z}+(1 / 2) u_{T T}+|u|^{2} u=0$, hence any solution to the latter equation generates an exact solution of the supersymmetric system, the most important one being the fundamental soliton with amplitude $\eta$,

$$
u_{\text {sol }}=\eta \exp \left(i \eta^{2} Z / 4\right) \operatorname{sech}(\eta T), v_{\text {sol }}=i u_{\text {sol }} .
$$

The linearization of Eqs. (2) around any exact solution of the form $v=i u$ leads to the following equations for small perturbations $\delta u$ and $\delta v$ :

$$
\mathcal{L}(\delta u+i \delta v)=0, \mathcal{L}(\delta u-i \delta v)=2 i \kappa(\delta u+i \delta v),
$$
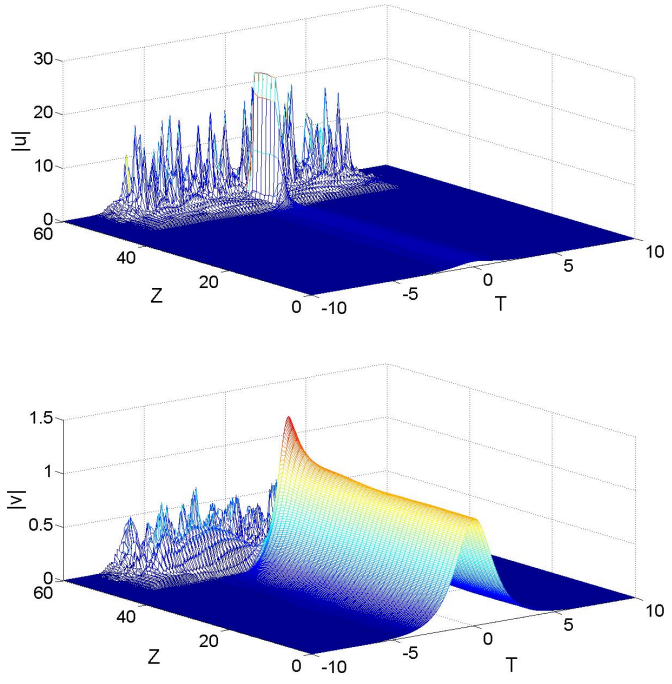

Fig. 1: (Color online) Instability of a fundamental soliton in the supersymmetric system (2) with $\kappa=1$. The solution is generated by the input corresponding to soliton (3) with a small perturbation, $u_{0}(T)=-i v_{0}(T)=1.03 \operatorname{sech}(T)$. Panels (a) and (b) display the evolution of the wave components in the amplified and damped cores, respectively (note the difference in the scales of the vertical axes between (a) and (b)).

where $\mathcal{L} \delta u \equiv\left[i \partial_{Z}+(1 / 2) \partial_{T T}+2|u|^{2}\right] \delta u+u^{2} \delta u^{*}$ corresponds to the linearization of the NLS equation. If $u(Z, T)$ is stable as the solution to the NLS equation (the fundamental soliton definitely is), the first equation in system (4) produces no instability, while a resonance is anticipated in the second, inhomogeneous, equation, as $\delta u+i \delta v$ is an eigenmode (corresponding to of the same operator $\mathcal{L}$ which figures on the right-hand side of equation. As follows from the commonly known linear-resonance theory, perturbations generated by the latter equation grow linearly in $Z$, rather than exponentially. Simulations of Eqs. 20 confirm that all the solitons are unstable, the character of the instability development being consistent with the subexponential growth, see Fig. 1

As said above, we aim to develop the management method for stabilizing solitons in the "supersymmetric" system, which amounts to periodically switching the gain and loss between the cores, with the simultaneous reversal of the sign of the coupling coefficient. The accordingly modified equations 2 are

$$
\begin{gathered}
i u_{Z}+(1 / 2) u_{T T}+|u|^{2} u \\
+\kappa \operatorname{sgn}\{\sin (2 \pi Z / L)\}(-i u+v)=0, \\
i v_{Z}+(1 / 2) v_{T T}+|v|^{2} v \\
+\kappa \operatorname{sgn}\{\sin (2 \pi Z / L)\}(i v+u)=0,
\end{gathered}
$$

where $L$ is the management period. We stress that the management does not break the supersymmetry of the system, and, accordingly, wave forms with $v(Z, T)=i u(T, v)$, 
where $u$ is any solution to the usual NLS equation, remain exact solutions of system (5).

The stabilizing effect of the management can be explained by its action on perturbations around the exact solutions. In particular, the replacement of constant $\kappa$ in the second equation in system (4) by the periodically flipping coefficient detunes the destabilizing resonance. The detuning is small for a large management period, hence the stabilization effect is expected to be weak in this case, which is indeed observed, see Fig. 3 below. Previously, effects of the periodic modulation of the coupling strength on the stability of solitons were studied in the model of the usual (conservative) dual-core systems 30,32. Those effects may be rather involved, but, generally speaking, they do not stabilize the symmetric solitons against the symmetry-breaking bifurcation, as rapid modulations effectively weaken the linear coupling between the cores, thus making the symmetric solitons less robust against the spontaneous symmetry breaking. Thus, the effects of the management mechanism acting on the "supersymmetric" solitons are different from the earlier studied ones.

The stabilization of solitons by means of the management. Simulations of Eqs. (5) reveal that the periodic application of the switching not only stabilizes the exact soliton solutions against small perturbations, but actually turns the solitons into strong attractors for inputs significantly different from the exact solutions. Typical examples illustrating the rapid convergence of the inputs, taken as

$$
u_{0}(T)=A \operatorname{sech}(T), v_{0}=i u_{0},
$$

with amplitudes $A$ differing by $+20 \%$ or $-10 \%$ from the value $A_{0}=1$ in the respective exact soliton solution (3), into solitons are displayed in Fig. 2. Note that the propagation distance shown here in units of $Z$ actually coincides, within the order of magnitude, with that measured in units of the soliton's dispersion length, therefore the propagation distance displayed in the figure is extremely large, making the full stabilization of the solitons obvious.

While the stabilization seems rather obvious in the case of the short-period management (as in that case the frequent switch between the loss and gain may nearly average them to zero), the model provides conspicuous stabilization even for very large management periods. The results of systematic simulations are summarized in Fig. 3, which displays stability domains (areas between the curves) in the plane of the management period $L$ and input amplitude $A$ (defined as per Eq. (6)), for different values of the coupling parameter $\kappa$ in Eqs. (5). While it is natural that the stability area expands with the decrease of $\kappa$ (which corresponds to weaker perturbations), it is worthy to note that the stability regions remain appreciable even for very strong coupling $(\kappa=10)$. Note that (as was mentioned above) the stability areas may be realized as attraction basins for the stable solitons which self-trap from input pulses (6).

Amplitude $B$ of the self-trapped soliton, that has the
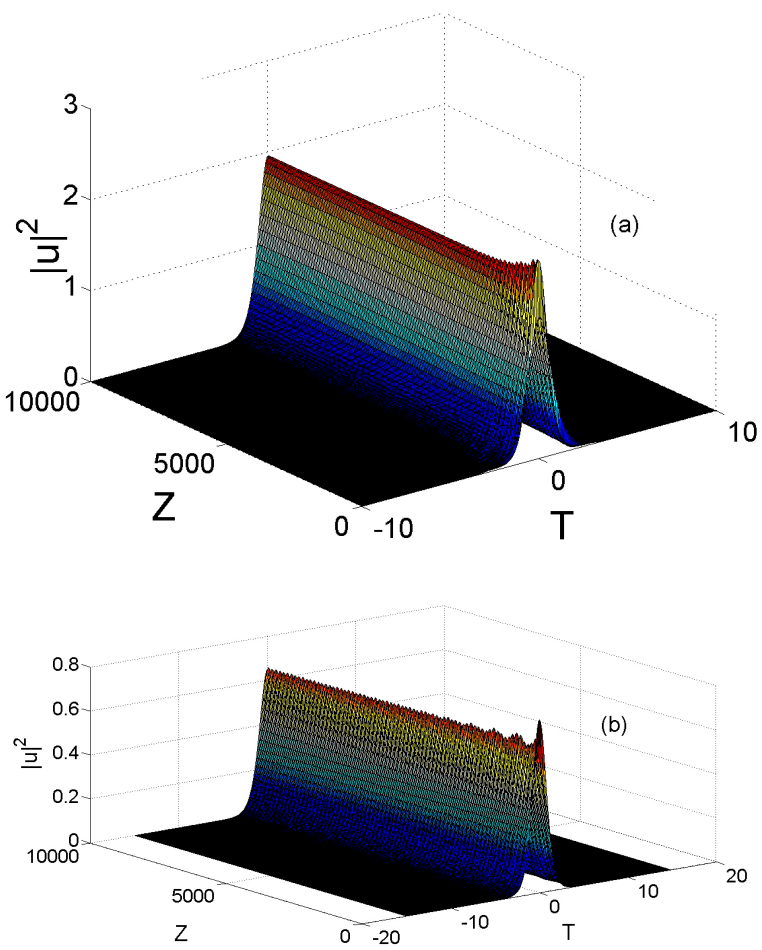

Fig. 2: (Color online) The self-trapping of inputs (6) with $A=$ 1.2 (a) and $A=0.9$ (b) into stable solitons, demonstrated by simulations of Eqs. (5) for $\kappa=1$ and $L=2$. The evolution of the $v$-component is similar to that displayed here for $u(Z, T)$.



Fig. 3: (Color online) The soliton stability regions in the $(L, A)$ plane for different values of coupling parameter $\kappa$ (see Eqs. (5) and (6)). The inset shows amplitude $B$ of the emerging stable soliton as a function of input amplitude $A$. 

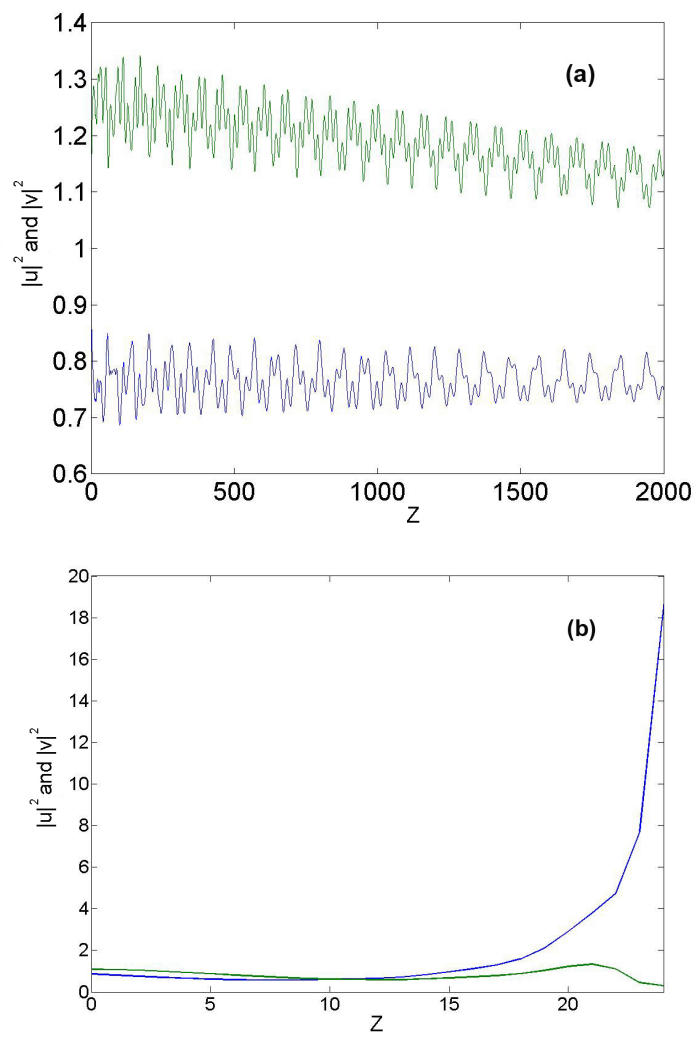

Fig. 4: (Color online) The evolution of the peak powers of both field components in the case of the initial conditions with asymmetric amplitudes, $u_{0}=1.05 \operatorname{sech}(T), v_{0}=0.95 i \operatorname{sech}(T)$. The coupling parameter is $\kappa=0.25$. (a) The system under the action of the management with period $L=2$. (b) The system without the management.

form of $|u(T)|=|v(T)|=B \operatorname{sech}(B T)$, is a function of the input's amplitude $A$ of initial condition (6), as shown in the inset of Fig. 3. It is worthy to note that, unlike the stability area, dependence $B(A)$ is weakly affected by the variation of $L$ and $\kappa$.

Obvious peculiarities of the exact supersymmetric solutions, with $v=i u$, are the equality of the amplitudes of the two components, and the fixed phase shift between them, $\pi / 2$. The use of inputs which break either of these relations impedes the self-trapping into exact solitons. In such a case, the management gives rise to overall-stable two-component solitary breathers, which feature persistent irregular oscillations and extremely slow evolution of the average peak power. A typical evolution history produced by the input with the asymmetry in amplitudes is illustrated by Fig. 4(a). For comparison, Fig. 4(b) shows that the same input pulse quickly collapses in the system without management.

The solitons in the supersymmetric system are also sensitive to a mismatch in the phase shift between the two components. The simulations with input

$$
u_{0}(T)=\operatorname{sech}(T), v_{0}=i \exp (i \phi) u_{0}(T),
$$
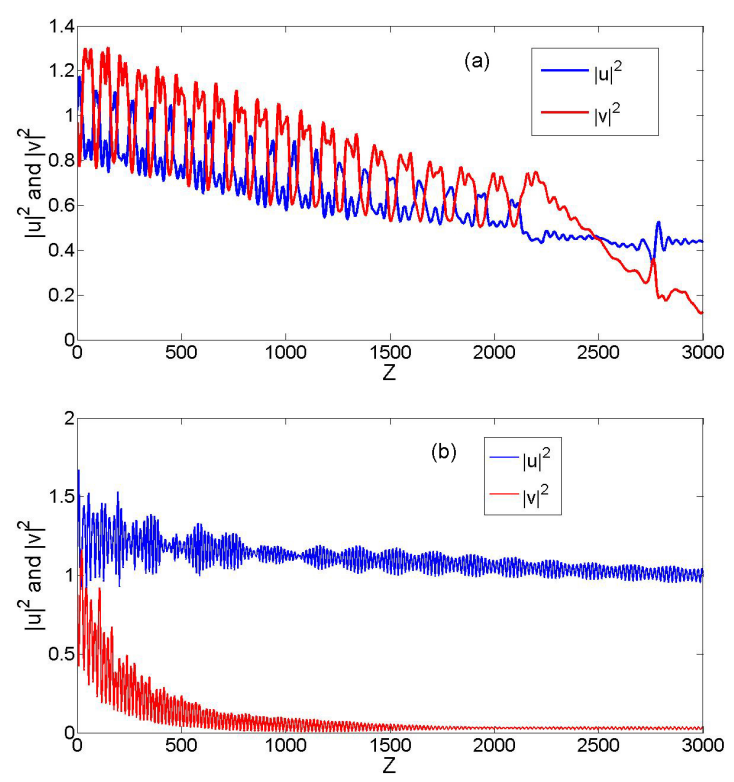

Fig. 5: (Color online) The same as in Fig. 4. but for $\kappa=$ 1 and initial condition (7) with phase mismatch between the components: $\phi=0.25 \pi$ in (a) and $\phi=0.5 \pi$ in (b).

where $\phi$ is the phase mismatch (cf. Eq. (3)), generate quasi-stable two-component breathers, which remain coupled over a finite but long propagation distance, if $\phi$ is not too large. An example for $\phi=0.25 \pi$ is displayed in Fig. 5 (a). On the other hand, the increase of $\phi$ in the same case to $\phi=0.5 \pi$ leads to quick decoupling of the components and destruction of the two-component soliton, as shown in Fig. 5(b).

Collecting results of the simulations, it is possible to identify a critical value of the mismatch, $\phi_{\mathrm{cr}}$, which separates the quasi-stable transmission of the two-component soliton and its quick splitting. Fixing $\kappa \equiv 1, \phi_{\text {cr }}$ is displayed as a function of the management period, $L$, in Fig. 6. It is observed that the phase mismatch produces the strong destabilization effect at small and large values of $L$, while a flat optimum region is identified at $1 \lesssim L \lesssim 2.5$. For $L<1$, detailed consideration of the numerical results explains the destabilization as follows: small $L$ affects, first of all, the coupling between the components, practically nullifying it due to the averaging over frequent flips of the sign of $\kappa$. Thus, the system splits into almost decoupled components, and the one which starts its evolution under the action of the loss cannot recover after the initial decay, despite the subsequent switch of the loss into the gain (similar to what is seen in Fig. 5(b)). In the opposite case of large $L$, the splitting of the components is simply explained by the fact that one of them suffers strong attenuation during the first period of the management.

Conclusion. We have introduced a system based on the dual-core coupler with the intrinsic Kerr nonlinearity, which features the supersymmetry, as an extended version of the $\mathcal{P} \mathcal{T}$ symmetry, with the gain and loss in the 


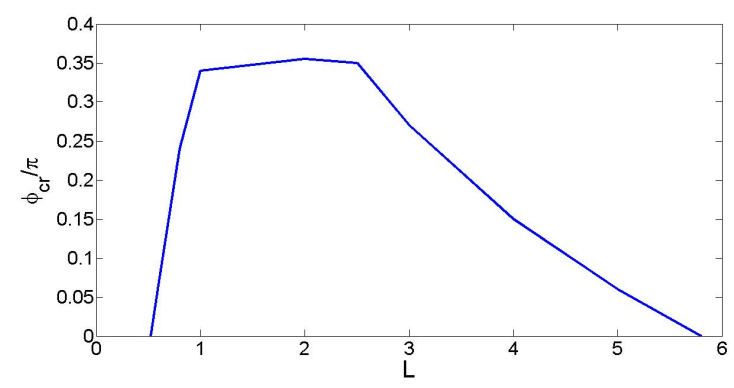

Fig. 6: (Color online) The critical value of the initial phase mismatch, $\phi_{\mathrm{cr}}$, for the input (7), versus the management period, $L$, for $\kappa=1$ in Eq. (5).

two cores equal to the strength of the inter-core coupling. The supersymmetry allows one to find a broad class of exact solutions, including solitons, for which the stability is a crucial issue. The exact solutions are vulnerable to the specific subexponential instability, which destroys all the solitons. We have demonstrated that the management, defined as periodic synchronous switch of the signs of the gain, loss, and linear coupling readily stabilizes the solitons, keeping the system's supersymmetry intact. Actually, the management makes solitons attractors, for which we have identified the attraction basins. The amplitude asymmetry and phase mismatch between the two components in the input pulse turns the stationary solitons into quasi-stable breathers. We have identified the stability area for the breather with respect to the phase mismatch and management period.

As an extension of this study, it may be relevant to consider interactions between the stabilized solitons. A challenging problem is to analyze two-dimensional doublecore supersymmetric systems.

\section{REFERENCES}

[1] C. M. Bender, Rep. Prog. Phys. 70 (2007) 947.

[2] A. Ruschhaupt, F. Delgado, and J. G. Muga, J. Phys. A 38 (2005) L171.

[3] R. El-Ganainy, K. G. Makris, D. N. Christodoulides, and Z. H. Musslimani, Opt. Lett. 32 (2007) 2632.

[4] M. V. Berry, J. Phys. A 41 (2008) 244007.

[5] K. G. Makris, R. El-Ganainy, D. N. Christodoulides, and Z. H. Musslimani, Phys. Rev. Lett. 100 (2008) 103904.

[6] S. Klaiman, U. Günther, and N. Moiseyev, Phys. Rev. Lett. 101 (2008) 080402.

[7] S. Longhi, Phys. Rev. Lett. 103 (2009) 123601.

[8] S. Longhi, Phys. Rev. A 82 (2010) 031801(R).

[9] Y. N. Joglekar, D. Scott, M. Babbey, and A. Saxena, Phys. Rev. A 82 (2010) 030103(R).

[10] A. Guo, G. J. Salamo, D. Duchesne, R. Morandotti, M. Volatier-Ravat, V. Aimez, G. A. Siviloglou, and D. N. Christodoulides, Phys. Rev. Lett. 103 (2009) 093902.

[11] C. E. Ruter, K. G. Makris, R. El-Ganainy, D. N. Christodoulides, M. Segev, and D. Kip, Nature Physics 6 (2010) 192.
[12] K. G. Makris, R. El-Ganainy, D. N. Christodoulides, and Z. H. Musslimani, Int, J. Theor. Phys. 50 (2011) 1019.

[13] A. A. Sukhorukov, Z. Xu, and Y. S. Kivshar, Phys. Rev. A 82 (2010) 043818.

[14] H. Ramezani, T. Kottos, R. El-Ganainy, and D. N. Christodoulides, Phys. Rev. A 82 (2010) 043803.

[15] K. Li and P. G. Kevrekidis, Phys. Rev. E 83 (2011) 066608.

[16] S. V. Dmitriev, S. V. Suchkov, A. A. Sukhorukov, and Y. S. Kivshar, Phys. Rev. A 84 (2011) 012123.

[17] F. Kh. Abdullaev, Y. V. Kartashov, V. V. Konotop, and D. A. Zezyulin, Phys. Rev. A 83 (2011) 041805(R).

[18] A. Miroshnichenko, B. A. Malomed, and Y. S. Kivshar, Phys. Rev. A, 84 (2011) 0121123(R).

[19] Z. H. Musslimani, K. G. Makris, R. El-Ganainy, and D. N. Christodoulides, Phys. Rev. Lett. 100 (2008) 030402.

[20] B. A. Malomed and H. G. Winful, Phys. Rev. E 53 (1996) 5365.

[21] J. Atai and B. A. Malomed, Phys. Rev. E 54 (1996) 4371.

[22] H. Sakaguchi and B. A. Malomed, Physica D 147 (2000) 273.

[23] W. J. Firth and P. V. Paulau, Eur. Phys. J. D 59 (2010) 13.

[24] C. Li, G. Xu, L. Ma, N. Dou, and H. Gu, J. Opt. A: Pure Appl. Opt. 7 (2005) 540.

[25] A. Marini, D. V. Skryabin, and B. A. Malomed, Opt. Exp. 19 (2011) 6616.

[26] B. A. Malomed, Chaos 17 (2007) 037117.

[27] P. V. Paulau, D. Gomila, T. Ackemann, N. A. Loiko, and W. J. Firth, Phys. Rev. E 78 (2008) 016212.

[28] P. V. Paulau, D. Gomila, P. Colet, N. A. Loiko, N. N. Rosanov, T. Ackemann, and W. J. Firth, Opt. Exp. 18 (2010) 8859.

[29] P. V. Paulau, D. Gomila, P. Colet, B. A. Malomed, and W. J. Firth, "From one- to two-dimensional solitons in the Ginzburg-Landau model of lasers with frequency-selective feedback", Phys. Rev. E, in press.

[30] P. L. Chu, B. A. Malomed, G. D. Peng, and I. M. Skinner, Phys. Rev. E 49 (1994) 5763.

[31] R. Driben, B. A. Malomed, and P. L. Chu, J. Opt. Soc. Am. B 20 (2003) 2338.

[32] B. A. Malomed, Soliton Management in Periodic Systems (Springer: New York, 2006). 